\title{
Protocol design and ideas research exchange \\ Exercise program on the health of people with epilepsy: protocol of a randomized controlled trial study
}

\author{
César Augusto Häfele ${ }^{1}$ (D) , Airton José Rombaldi ${ }^{1}$ (1) , Vitor Häfele ${ }^{1}$ (D) , Lucas Rossetto ${ }^{2}$ (D) , Natan Feter ${ }^{1}$ (D) , \\ Bianca Lamas Gervini $^{4}$ (D) , Thiago Terra Borges ${ }^{3}$ (D) , Eduardo Lucia Caputo ${ }^{1}$ (D) , Paola Leal de Oliveira ${ }^{5}$ (D) , \\ Steve Anthony Maravillo ${ }^{6}$ (i) , Marcelo Cozzensa da Silva ${ }^{1}$ \\ ${ }^{1}$ Universidade Federal de Pelotas, Escola Superior de Educação Física, Pelotas, RS, Brasil; ${ }^{2}$ Universidade \\ Federal de Pelotas, Faculdade de Medicina, Pelotas, RS, Brasil; ${ }^{3}$ Instituto Federal de Educação, Ciência e \\ Tecnologia Sul-rio-grandense, Pelotas, RS, Brasil;; ${ }^{4}$ Universidade Federal de Pelotas, Clinica de Neurologia da \\ Faculdade de Medicina, Pelotas, RS, Brasil; ' Universidade Federal de Pelotas, Faculdade de Psicologia, \\ Pelotas, RS, Brasil, ${ }^{6}$ University of California San Diego, Public Health School, San Diego, CA, United States.
}

Associate Editor: Iane de Paiva Novaes, UESB/Jequié, BA, Brazil.

\begin{abstract}
Aim: This study aimed to describe an experimental protocol to evaluate how a structured exercise program can impact on the health of people with epilepsy. Methods: For this purpose, a randomized control trial will be conducted in the city of Pelotas, southern Brazil. Participants will be randomized into two groups: 1) Control- maintain usual activities, and 2) Exercise- a structured exercise program, with two 60-minute sessions per week, for 12 weeks. The intervention will be composed by warm-up (5-minutes), moderate-intensity aerobic exercise (15-20 minutes, performed on a treadmill), resistance training (2-3 sets, 10-15 repetitions), and 5-minutes of stretching exercises. Sociodemographic characteristics, clinical information, cognitive function, anthropometric measurements (weight, height, hip and waist circumferences), cardiorespiratory fitness $\left(\mathrm{VO}_{2} \mathrm{max}\right.$ ), and strength (dynamometer) will be measured at baseline and 12-week post-intervention. Participants will receive a diary to record their seizures throughout the study. Results: A structured exercise program is expected to generate beneficial health effects, and the results can assist in health professionals' clinical practice for people with epilepsy.
\end{abstract}

Keywords: epilepsy; motor activity; seizures; trial; mental health.

\section{Introduction}

Epilepsy is one of the most prevalent neurological diseases, especially in low- and middle-income countries, ${ }^{1}$ that affects nearly 50 million people of all ages worldwide ${ }^{2}$. Epilepsy is characterized by hyperexcitability in the brain that leads to recurrent epileptic seizures. Although the cause of the disease is still unknown in about $50 \%$ of global cases, many underlying disease mechanisms can lead to epilepsy ${ }^{2}$. This disease is characterized by a brain disorder defined by any of the following conditions: a) at least two unprovoked epileptic seizures within 24 hours; b) an unprovoked seizure, and a high risk of having another seizure; or c) a diagnosis of epileptic syndrome ${ }^{3}$. The World Health Organization estimates that only $25 \%$ of epileptic patients have access to proper treatment and $70 \%$ could live seizure-free if properly diagnosed and treated ${ }^{2}$.

This disease is a chronic condition that directly affects psychological, social, and labor factors among individuals ${ }^{4}$. These people are more likely to present psychiatric morbidities, such as anxiety and depression ${ }^{5}$, and may also present cognitive impairment in the domains of learning, memory, attention, and executive functions ${ }^{6,7}$. Antiepileptic drugs can exacerbate the deficits arising from this neurological disease. ${ }^{8}$ Although carbamazepine was associated with deficits in attention ${ }^{9}$ and verbal fluency, ${ }^{10}$ valproate and phenobarbital were associated with worse cognitive deficit ${ }^{11,12}$. Together, these factors portray a negative influence on the quality of life among this population ${ }^{13,14}$.

In addition to conventional treatment, non-pharmacological therapies have been used to treat epilepsy. Regular exercise is an emerging strategy for improving health conditions, seizure control, and cognitive function in people with epilepsy $y^{15-17}$. Studies have shown that exercise might have a neuroprotective action in the brain, ${ }^{18}$ protecting against seizures ${ }^{17,19}$. Some studies have assessed the chronic effect of exercise on people with epilepsy ${ }^{19-21}$. Erikssen et al. ${ }^{19}$ showed that physical exercise may also contribute to control epileptic seizures. McAuley et al. ${ }^{20}$ and Nakken et al. ${ }^{21}$ did not find differences in the frequency of seizures, however, an improvement in health and psychological variables was reported.

There are few studies worldwide that evaluate the chronic effect of physical exercise on the health of people with epilepsy ${ }^{21}$. Therefore, promotion of physical activity or exercise could be an emergent low-cost nonpharmacological approach to reduce the burden of epilepsy for the patients, mainly in low- and middle-income countries where approximately $80 \%$ of people with epilepsy are living ${ }^{2}$. Thus, this study aims to present an experimental protocol to evaluate how a structured exercise program can impact the health of people with epilepsy. 


\section{Methods}

This study will be a randomized controlled trial, single-blinded, conducted in the city of Pelotas, southern Brazil. The study protocol was approved by the Ethics Committee of the School of Physical Education from the Federal University of Pelotas (protocol number: 2.187.723). The clinical trial has been registered at www.ensaiosclinicos. gov.br (registration number: RBR-8h4dty).

In addition, the ethical procedures will be followed: (1) participation in the study will occur only after consent form has been signed; (2) data confidentiality will be guaranteed; (3) participants reporting health problems, after or during the study, will be referred to appropriate health services.

\section{Participants recruitment}

People with epilepsy who used the Brazilian Public Health System primary care service, who are possibly in greater social vulnerability, will be invited first. Participants must be diagnosed with epilepsy (confirmed by a physician who will be part of the research team) and be on antiepileptic drugs. The recruitment will be as follow: 1) people with epilepsy from the Neurology Outpatient Clinic of the Medical School - Federal University of Pelotas, participants from a previous descriptive study, ${ }^{22} 2$ ) identification on records from the Neurology Outpatient Clinic of the Medical School - Federal University of Pelotas; and 3) dissemination by social media (e.g. Facebook, WhatsApp) and local press.

\section{Participants}

Potential participants who agree to take part in the study will be instructed to attend the School of Physical Education in the following week to receive detailed explanations and sign the consent form. To be included in the study participants must comply with the following characteristics: 1) age between 18 and 60 years; 2) sedentary; 3) and diagnosed with epilepsy (confirmed by a physician). Physical impairment that does not allow exercise practice or inability to answer the questionnaire, will be defined as exclusion criteria. All participants will be required to undergo a physical examination performed by a physician to ensure safety to engage in exercise training. The participants with uncontrolled blood pressure will be instructed to look for a health center.

Participants will be randomized into two groups: exercise (EG) or control group (CG). EG will perform two 60-minute sessions per week of combined training (aerobic + strength + stretching), for 12 weeks. The CG will be recommended to maintain their usual daily activities. To ensure equivalence in sample size between groups the block randomization technique, with a 1:1 allocation rate will be used. In each block, the first patient drawn will be allocated to EG, and the second to $\mathrm{CG}$, and so on.

\section{Intervention}

The intervention will take place at the gym of the School of Physical Education of the Federal University of Pelotas, which will supply treadmills, bicycles, dumbbells, shin guards, elastic bands, Swiss balls, and weight-training equipment for strength training, in addition to an air-conditioned environment. The schedules training will be from 6 a.m. to 9 p.m. from Monday to Friday, and from 9 a.m. to 5 p.m. on Saturdays. All training sessions will be individually guided by a trained exercise science professional. In addition, door-to-door transportation to and from the gym will be provided for research participants.

In case of absence, another day will be offered to recover training and a $70 \%$ adherence rate will be required to be included in the analysis. Participants will be instructed not to exercise two days in a row.

While participants allocated to the CG will be encouraged to continue their normal daily activities, the participants in EG will receive a structured, individually supervised, exercise program with two 60-minute sessions per week. Each session will be composed of warm-up, aerobic, strength, and stretching exercises. The periodization and exercise program were adapted from other studies ${ }^{20,23,24}$ and will be based on the American College of Sports Medicine recommendations for sedentary people ${ }^{25}$. The exercise program will be divided into three cycles, each cycle performed in one month, using progressive periodization. Physical tests will be monitored by two exercise science specialists and a physician. All professionals will be trained on safety measures in case a seizure occurs.

Warm-up will be performed at the first 5 minutes of the sessions, using light activity (walking at treadmill). Immediately after warm-up, aerobic training will be started at a treadmill, with intensity controlled by the rating of perceived exertion (RPE) using the 6-to-20 Borg scale ${ }^{26}$ with target intensity between 14 and 17.

Resistance training will be focused on main muscle groups using strength exercise machines and free weights. The following exercises will be performed: chest press, seated row, leg press, squat, shoulder press, sit-up, and plank. During the last 5 minutes of each session, active stretching (shoulders, hamstrings, quadriceps, chest, triceps, lower back, and neck) will be executed in 3 sets of 10 seconds in each exercise.

From week 1 to 4 , each session will begin with a 5-minute warm-up, 15 minutes of aerobic exercise, 35 minutes of strength training, and 5 minutes of stretching. In the first week, there will be an adaptation period to emphasize the correct execution of movements, in which two series of 12 to 15 submaximal repetitions will be performed.

In weeks two, three, and four, the intensity will be increased and participants will be instructed to perform 12 to 15 maximum repetitions. Then, at weeks five to eight, the volume will be increased in aerobic (20 minutes) and strength training (3 sets). Lastly, at week nine to twelve, participants will perform 25 minutes of aerobic training and 3 sets of 10 to 12 maximum repetitions at strength training. The total time on plank will be increased from 20 seconds from weeks 1 to 4 , to 30 seconds at weeks 5 to 8 , and then 45 seconds from weeks 9 to 12 . The 
interval time will always be 90 seconds between sets ${ }^{24}$ and as suggested by the American College of Sports Medicine, ${ }^{25}$ the intensity will be controlled by a trained exercise Science based on evaluation of perceived exertion (Figure 1).

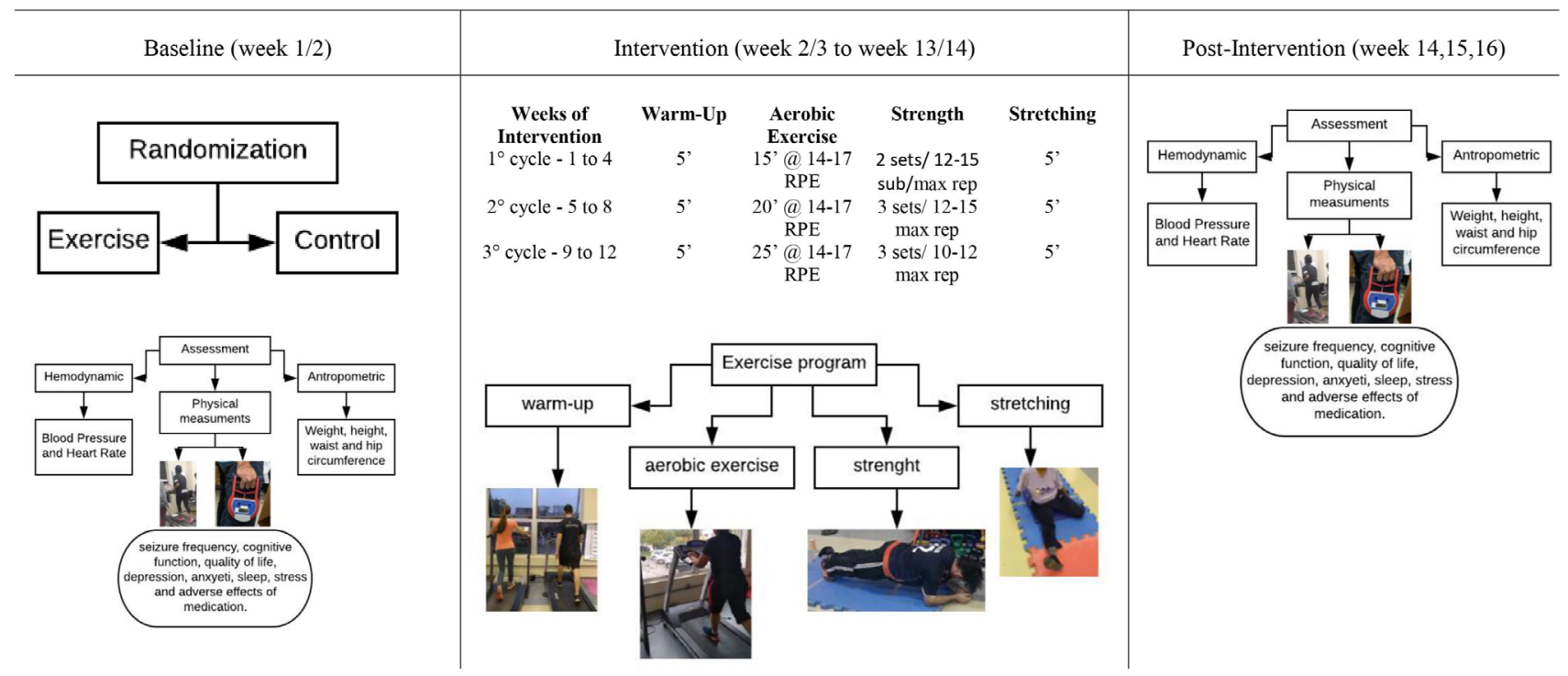

Figure 1 - Overview of the protocol.

Max rep: maximal repetition; Sub: submaximal repetition; RPE: rating of perceived exertion.

Assessments

\section{Baseline}

Baseline measures will be held in two visits. In the first visit, participants will answer a questionnaire covering sociodemographic, neuropsychological, clinical, and health questions. In addition, a strength test, as well as a treadmill and the mask used for the maximum oxygen consumption $\left(\mathrm{VO}_{2} \max \right)$ test familiarization will be conducted. In the second visit, hemodynamic (blood pressure and heart rate), anthropometric (weight, height, waist, and hip circumferences), and $\mathrm{VO}_{2}$ max test (accompanied by a physician) will be performed. In addition, a seizure diary will be provided, in which subjects will record the days and the number of seizures throughout the trial.

\section{Primary outcomes}

\section{Frequency and Number of Epileptic Seizures}

Participants will be asked, in the baseline, about the seizure experience: "Have you had seizure in the past three months?". If yes, they will be asked about the number of seizures: "How many seizures did you have?".

\section{Quality of Life}

Quality of Life in Epilepsy Inventory (QOLIE-31) will be used. This instrument contains 31 items divided into seven domains: seizure worry, the general quality of life, emotional well-being, energy and fatigue, cognitive function, social function, and medication effects. The scores range from 0 to 100 , with results closer to 100 corresponding to a better quality of life ${ }^{27}$.

\section{Depression}

The Neurological Disorders Depression Inventory for Epilepsy (NDDI-E) will be used. It contains six items and its final score can vary from 6 to 24. Scores higher than or equal to 15 indicate a diagnosis of depression ${ }^{28}$.

\section{Anxiety}

The short version of the State-Trait Anxiety Inventory (STAI) will be used for this study. Each sub-scale contains six items, totaling 12 questions for anxiety assessment. The anxiety state scale describes the feelings of the individual at a given time, under specific conditions. The trait anxiety scale describes the subjects' usual feelings. The scores, both for trait and anxiety state, range from six to 24 points. The higher scores, the higher state, and trait of anxiety ${ }^{29}$. 


\section{Adverse Effects of Antiepileptics}

The Adverse Effects Profile (AEP) will be used to evaluate the adverse effects of antiepileptic drugs. The scale has 19 items and scores vary between 19 and 76 . The higher the scores, the higher the perceived adverse effects ${ }^{30}$.

\section{Cognitive Function}

\section{Global Cognitive Function}

The Montreal Cognitive Assessment (MOCA) will be used to assess cognitive function. This instrument evaluates visuospatial and executive function, appointment, memory, attention, language, late evocation, and orientation. Scores range from 0 to 30 points $^{31}$. In addition, the trail making test, digit span test, the Stroop color, and word test, and verbal fluency test will be used in order to assess mental flexibility, working memory, inhibit cognitive interference, and verbal fluency, respectively.

\section{Attention, visual search speed, speed of processing, mental flexibility, and executive function}

Trail making test will provide valuable information about visual search speed, speed of processing, mental flexibility, and executive function ${ }^{32}$. In the first moment, a researcher will present a figure with 8 circles with numbers from 1 to 8 inside each one. The researcher will explain that the participant should draw a line from number 1 (point to "1") to number 2 (point to "2"), 2 to 3 (point to " 3 "), and so on. After this non-scored task, the researcher will present a second figure with 25 numbers (1-25), and the participants should follow the same instruction that the previous task. Later, the researcher will show to the participant a figure with 8 circles with numbers (1-4) or letters (A to D) inside each circle. The researcher will give the following instruction: draw a line from number 1 (point to "1") to letter A (point to "A"), A to 2 (point to "2"), 2 to B (point to "B") and so on. In the last task from this test, participants will be given a figure with 25 circles with numbers (1-13) or letters (A to L) inside each circle. The researcher will give instructions as follow: draw a line from number 1 (point to " 1 ") to letter A (point to "A"), A to 2 (point to "2"), 2 to B (point to "B") and so on. In all tasks, the participants will be instructed not to lift the pencil from the paper. The time spent in each task will be recorded.

\section{Working memory}

Digit span test will be used to identify working memory from participants. ${ }^{33}$ In the first part of this test, a sequence of numbers will be presented to them, starting from sequences compounded from 2 to 7 digits. Immediately after the researcher says the first sequence, participants should recall the same digits at the same sequence. If participants do not recall the information correctly, the researcher will give a new sequence with the same number of digits. In case they recall incorrectly again, this part of the test will be ended. If not, the researcher will give a new sequence compounded by 3 digits. The test will end when participants make two consecutive errors. In the second part, the instruction will be the same as previously, however, participants should recall the numbers backward.

\section{Cognitive interference}

Stroop color and word task tests the inhibit cognitive interference, an important compound of executive function ${ }^{34}$. Participants should read a list of 112 words ignoring the printed color of each word. In the second moment, participants should name the color from which each word was printed ignoring its meaning. The time to complete the list in each task will be recorded.

\section{Language domain}

A verbal fluency task will be performed to assess another domain of executive function ${ }^{35}$. Participants will be asked to recall in one minute, as many words they can, that start with letters F, A, and S. Words repetition, proper names, and words derived in number, gender, conjugation, and degree of comparison will not be scored. Fluency will be indexed by the sum of correct words across all three trials.

\section{Stress}

The Perceived stress scale (PSS-10) will be used. This scale is composed of 10 items and refers to situations and events of the last 30 days. Each item has a Likert scale that varies from 0 (never) to 4 (very frequent). Scores range from 0 to 40 , and the higher the score, the higher the individual's perception of stress ${ }^{36}$.

\section{Quality of Sleep}

The Pittsburg Sleep Quality Index (PSQI) will be used..$^{37}$ The questionnaire contains 19 items and for each item, a scale from 0 to 3 will be used. The total score ranges from 0 to 21 , with scores between 0 and 4 indicating good sleep quality, 5 to 10 indicating bad quality, and above 10 indicating a sleep disorder.

\section{Secondary outcomes}

\section{Strength}

The handgrip strength test will be conducted, using a Takei Scientific Instruments dynamometer, model T.K.K.5401. The 
participant will be standing, with their feet together, arms close to the body, elbows extended, and holding the dynamometer with the dominant hand. When the researcher gives a signal, the participant will have three seconds to tighten the device. Three attempts will be made with an interval of 30 seconds of rest among them and the highest value will be used.

\section{Physical fitness}

The $\mathrm{VO}_{2}$ max will be assessed using a maximum incremental protocol on a treadmill through a stress test, where at the end of each stage, $1 \mathrm{~km} /$ hour will be added to the running speed until volitively fatigue is reached. The initial test speed will be $6 \mathrm{~km} . \mathrm{h}$ ${ }^{1}$, and prior to that, a 4-minute warm-up will be performed at $6 \mathrm{~km} . \mathrm{h}^{-1}$. The gas collection will be performed by open-circuit spirometry, continuously, using the VO2000 (MedGraphics ${ }^{\circledR}$, Ann Arbor, USA) mix box type. The analyzer will be calibrated before each collection with ambient air, according to the manufacturer's recommendations and the subjects will be submitted to familiarization with the mask. Blood pressure will be checked using the Littmann Master Classic stethoscope and the Nylon Bic Sphygmomanometer and Contact Closure, before and after the test by a physician. The heart rate will be measured using a heart rate monitors (Polar ${ }^{\circledR}$ RS800CX, Finland) with a recording frequency of 1 beat per second; participants will use the device throughout the test. During the test, the intensity will be controlled by the objective (evaluator) and subjective (individual) perception of exertion by the Borg scale from 6 to $20^{26}$.

\section{Anthropometric}

Height and weight will be measured using a wall stadiometer (resolution scale of 0.1 centimeters) and a Filizola digital precision scale (resolution of $0.1 \mathrm{~kg}$ ), respectively. The waist circumference will be measured with an inextensible tape at the largest circumference between the last rib and the iliac crest. Hip circumference will be measured at the maximum protruding part of the buttocks at the level of the greater trochanter with the participant wearing minimal clothing and standing with feet together. In both, the waist and hip circumference measurements will be performed with minimal clothing while standing.

\section{Clinical variables}

The variables of interest related to epilepsy are type of epilepsy, antiepileptic drugs, type and duration of treatment (monotherapy or polytherapy, years with epilepsy, seizure control (controlled, not always controlled or not controlled) and clinical family history.

\section{Sample size and statistical analysis}

The sample size of this study is based on the expected difference in the primary outcome variable (quality of life) between the EG and the CG after 3 months. The sample size calculation was based on the difference of means, considering $80 \%$ statistical power and 5\% significance level. We have estimated that 20 people will be necessary for the study ${ }^{22}$.

The SPSS 20.0 software will be used to analyze the data. A p-value of $<0.05$ will be considered for statistical significance. The analysis will be conducted by treatment intent and by adherence to study protocol. Baseline characteristics will be presented using descriptive statistics to compare both groups. Data will be presented as mean (standard deviation), or frequency (\%), as appropriate. The Shapiro-Wilk test will be used to check for normality. For categorical variables, Fisher's exact test will be used (n, \%). Generalized Estimating Equations (GEE) or two-way analysis of variance with repeated measures (ANOVA two-way) will be used to compare moments (pre- and post-training) and groups (EG and CG). If the significant influence of any factors (group, time, group $\mathrm{x}$ time interaction) is found, differences between specific means will be verified by Bonferroni post-hoc test.

\section{Discussion}

Physical exercise has increasingly attracted attention as a non-pharmacological alternative to assist people with epilepsy treatment. Although the literature shows promising results, physical activity prevalence among people with epilepsy is $l o w^{22,38}$.

Studies evaluating the influence of physical exercise by using animal models of epilepsy showed important results. ${ }^{38-41}$ However, intervention research using physical exercise in humans is scarce ${ }^{21}$ with only one randomized clinical trial being found to date ${ }^{20}$.

A strength of our study is the fact that is going to be performed in an Upper-middle income country. In addition, the initial invitation for people who use the Public Health System, which is possibly in greater social vulnerability, is another strength of our study. To ensure a greater participation in the exercise program, free transportation to the intervention site and from intervention site to participant's home will be offered.

This is the first randomized clinical trial in Brazil and the second in the world to evaluate the impact of a structured exercise program on the health of people with epilepsy. If the trial hypothesis is confirmed, the results are expected to assist in clinical practice as a means of disease control and general improvement of the health conditions of these people.

\section{References}

1. Beghi E, Giussani G, Abd-Allah F, Abdela J, Abdelalim A, Abraha HN, et al. Global, regional, and national burden of epilepsy, 1990-2016: a systematic analysis for the Global Burden of Disease Study 2016. Lancet Neurol. 2019;18:357-75.

2. Epilepsy: a public health imperative: summary. Geneva, Switzerland: World Health Organization; 2019.

3. Fisher RS, Acevedo C, Arzimanoglou A, Bogacz A, Cross JH, Elger CE, et al. ILAE official report: a practical clinical definition of epilepsy, Epilepsia. 2014;55:475-482. 
4. Gilliam F. Optimizing health outcomes in active epilepsy, Neurology. 2002;58:9-19.

5. Scott AJ, Sharpe L, Hunt C, Gandy M. Anxiety and depressive disorders in people with epilepsy: a metaanalysis. Epilepsia. 2017;58(6):973-82.

6. Black LC, Schefft BK, Howe SR, Szaflarski JP, Yep HS, Privitera MD. The effect of seizures on working memory and executive functioning performance, Epilepsy Behav. 2010;17:412-419.

7. Leeman-markowski BA, Schachter SC. Treatment of cognitive deficits in epilepsy, Neurologic clinics. 2016;34;183-204.

8. Greener M. Beyond seizures: understanding cognitive deficits in epilepsy. Prog Neurol Psychiatry. 2013;17:31-2.

9. Wesnes KA, Edgar C, Dean ADP, Wroe SJ. The cognitive and psychomotor effects of remacemide and carbamazepine in newly diagnosed epilepsy. Epilepsy Behav. 2009;14:522-8.

10. Äikiä M, Jutila L, Salmenperä T, Mervaala E, Kälviäinen R. Long term effects of tiagabine monotherapy on cognition and mood in adult patients with chronic partial epilepsy. Epilepsy Behav. 2006;8:750-5.

11. Gillham RA, Read CL, McKee PJW, Larkin JG, Brodie MJ. Cognitive function in adult epileptic patients on long-term sodium valproate. J Epilepsy. 1991;4:205-10.

12. Calandre EP, Dominguez-Granados R, Gomez-Rubio M, Molina-Font JA. Cognitive effects of long-term treatment with phenobarbital and valproic acid in school children. Acta Neurol Scand. 1990;81:504-6.

13. Cramer JA, Blum D, Reed M, Fanning K. The influence of comorbid depression on quality of life for people with epilepsy, Epilepsy Behav. 2003;4:515-521.

14. Loring DW, Meador KJ, Lee GP. Determinants of quality of life in epilepsy, Epilepsy Behav. 2004;5:976-980.

15. Capovilla G, Kaufman KR, Perucca E, Moshé SL, Arida RM. Epilepsy, seizures, physical exercise, and sports: a report from the ILAE Task Force on Sports and Epilepsy, Epilepsia. 2016;57:6-12.

16. Allendorfer JB, Arida RM. Role of Physical Activity and Exercise in Alleviating Cognitive Impairment in People with Epilepsy, Clin Ther. 2018;40:26-34.

17. Vancini RL, de Lira CAB, Scorza FA, de Albuquerque M, Sousa BS, de Lima C, et al. Cardiorespiratory and electroencephalographic responses to exhaustive acute physical exercise in people with temporal lobe epilepsy. 2010;19:504-508.

18. Feter N, Penny JC, Freitas MP, Rombaldi AJ. Effect of physical exercise on hippocampal volume in adults: Systematic review and meta-analysis, Science \& Sports. 2018;33:327-338.

19. Eriksen HR, Ellertsen B, Grønningsaeter H, Nakken KO, Løyning Y, Ursin K. Physical exercise in women with intractable epilepsy, Epilepsia. 1994;35:1256-1264.

20. McAuley JW, Long L, Heise J, Kirby T, Buckworth J, Lehman $\mathrm{KJ}$, et al. A prospective evaluation of the effects of a 12-week outpatient exercise program on clinical and behavioral outcomes in patients with epilepsy, Epilepsy Behav. 2001;2:592-600.

21. Häfele CA, Freitas MP, Rombaldi AJ. Physical exercise effects on epilepsy in humans: a systematic review, Rev Neuroscien. 2015;23:328-336.

22. Häfele CA, Freitas MP, Gervini BL, Carvalho RM, Rombaldi AJ. Who are the individuals diagnosed with epilepsy using the Public
Health System in the city of Pelotas, southern Brazil? Epilepsy Behav. 2018;78:84-90.

23. Garber CE, Blissmer B, Deschenes MR, Franklin BA, Lamonte MJ, Lee I-Min, et al. Quantity and quality of exercise for developing and maintaining cardiorespiratory, musculoskeletal, and neuromotor fitness in apparently healthy adults: Guidance for prescribing exercise, Med Sci Sports Exerc. 2011;43:1334-1359.

24. Domingues MR, Bassani DG, Da silva SG, Coll CVN, Da silva BGC, Hallal PC. Physical activity during pregnancy and maternalchild health (PAMELA): study protocol for a randomized controlled trial, Trials. 2015;24:216-227.

25. ACSM AC of S. ACSM's guidelines for exercise testing and prescription. Lippincott Williams \& Wilkins; 2013.

26. Borg G. Perceived exertion as an indicator of somatic stress, Scand J Rehabil Med. 1970;2:92-98.

27. Silva TI, Marques CM, Alonso NB, Azevedo AM, WestphalGuitti AC, Caboclo LOSF, et al. Translation and cross-cultural adaptation of the Quality of Life in Epilepsy-31 (QOLIE-31), J Epilepsy Clin Neurophysiol. 2006;12:107-110.

28. Gilliam FG, Barry JJ, Hermann BP, Meador KJ, Vahle V, Kanner AM. Rapid detection of major depression in epilepsy: a multicentre study, Lancet Neurol. 2006;5:399-405.

29. Fioravanti-Bastos ACM, Cheniaux E, Landeira-Fernandez J. Development and validation of a short-form version of the Brazilian State-Trait Anxiety Inventory, Psicol: Reflex Crit. 2011;24:485-494.

30. Gilliam FG, Fessler AJ, Baker G, Vahle V, Carter J, Attarian H. Systematic screening allows reduction of adverse antiepileptic drug effects: a randomized trial, Neurology. 2004;62:23-27.

31. Nasreddine ZS, Phillips NA, Bedirian V, Charbonneau S, Whitehead V, Collin T, et al. The montreal cognitive assessment, MOCA: A brief screening tool formild cognitive impairment, J Am Geriatr Soc. 2005;53:695-699.

32. Bowie CR, Harvey PD. Administration and interpretation of the Trail Making Test, Nature protocols. 2006;1:2277-2281.

33. Raiford SE, Coalson DL, Saklofske DH, Weiss LG. Practical issues in WAIS-IV administration and scoring, In: Weiss LG, Saklofske DH, Coalson D, Raiford SE (Eds.), WAIS-IV: Clinical use and interpretation, Elsevier, New York, 2010, pp.25-60.

34. Scarpina F, Tagini S. The Stroop Color and Word Test, Front Psychol. 2017;8:557.

35. Williams LM, Simms E, Clark CR, Paul RH, Rowe D, Gordon E. The test-retest reliability of a standardized neurocognitive and neurophysiological test battery: "Neuromarker", Int. J. Neurosci. 2005,115:1605-1630.

36. Reis RS, Hino AA, Añez CR. Perceived stress scale: reliability and validity study in Brazil, J Health Psychol. 2010;15:107-114.

37. Bertolazi AN. Tradução, adaptação cultural e validação de dois instrumentos de avaliação do sono: Escala de Sonolência de Epworth e Índice de Qualidade do Sono Pittsburgh [Dissertação]. Porto Alegre: Faculdade de Medicina, Universidade Federal do Rio Grande do Sul. 2008.

38. Han K, Choi-Kwon S, Lee S. Leisure time physical activity in patients with epilepsy in Seoul, South Korea, Epilepsy Behav. 2011;20:321-325. 
39. Lin XY, Cui Y, Wang L, Chen W. Chronic exercise buffers the cognitive dysfunction and decreases the susceptibility to seizures in PTZ-treated rats, Epilepsy Behav. 2019;98:173-187.

40. Arida RM, Vieira AJ, Cavalheiro EA. Effect of physical exercise on kindling development, Epilepsy Res. 1998;30:127-132.

41. Arida RM, Scorza FA, Dos Santos NF, Peres CA, Cavalheiro EA. Effect of physical exercise on seizure occurrence in a model of temporal lobe epilepsy in rats, Epilepsy Res. 1999;37:45-52.

42. Häfele CA, Freitas MP, Silva MC, Rombaldi AJ. Are physical activity levels associated with better health outcomes in people with epilepsy?, Epilepsy Behav. 2017;72:28-34.

\section{Acknowledgments}

The authors would like to acknowledge the subjects who volunteered their time to participate in this study. This study was financed in part by the Federal Institute of Education, Science, and Technology South Rio Grande (IFSul). This study was financed in part by the Coordenação de Aperfeiçoamento de Pessoal de Nível Superior. - Brasil (CAPES) - Finance Code 001. "This study was financed in part by the Federal University of Pelotas (UFPel) - PIB-M/D Program”

\section{Corresponding author}

César Augusto Häfele

Universidade Federal de Pelotas, Escola Superior de Educação Física. Rua Luís de Camões, 625 .

96055630, Pelotas, RS, Brasil. Telephone: +55 (53) 981256483

Email: hafele.c@hotmail.com

Manuscript received on August 28, 2020

Manuscript accepted on September 18, 2020

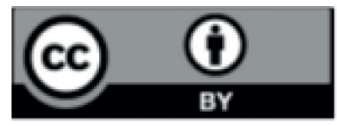

Motriz. The Journal of Physical Education. UNESP. Rio Claro, SP, Brazil - eISSN: 1980-6574 - under a license Creative Commons - Version 4.0 